\title{
5 Research Square

\section{Gene networks and canonical pathways associated with a favorable blood pressure response after CPAP treatment: the HIPARCO score post hoc analysis}

\section{Andrea Zapater \\ Institut de Recerca Biomedica \\ Fernando Santamaria-Martos \\ Institut de Recerca Biomedica \\ Adriano Targa \\ Institut de Recerca Biomedica \\ Lucía Pinilla \\ Institut de Recerca Biomedica \\ Alicia Sánchez-de-la-Torre \\ Institut de Recerca Biomedica \\ Ivan Benitez \\ Institut de Recerca Biomedica}

Miguel Angel Martinez

Hospital Universitari i Politecnic La Fe

\section{Ferran Barbé}

Hospital Universitari Arnau de Vilanova

Manuel Sánchez-De-La-Torre ( $\sim$ sanchezdelatorre@gmail.com )

IRB Lleida

\section{Research}

Keywords: microRNAs; obstructive sleep apnea; resistant hypertension; cardiovascular diseases; CPAP.

Posted Date: May 21st, 2020

DOI: https://doi.org/10.21203/rs.3.rs-29525/v1

License: (c) (i) This work is licensed under a Creative Commons Attribution 4.0 International License.

Read Full License 


\section{Abstract}

Background: Several studies report an association between microRNAs (miRNAs) and hypertension or cardiovascular disease. In a previous study, we observed a cluster of three miRNAs (miR-378a-3p, miR100-5p, and miR-486-5p) functionally associated with the cardiovascular system that predicted a favorable blood pressure response to continuous positive airway pressure (CPAP) treatment in patients with resistant hypertension (RH) and obstructive sleep apnea (OSA) (HIPARCO score). However, little is known regarding the molecular mechanisms underlying this. Thus, the aim of the present study was to perform a post hoc analysis to investigate the genes, functions and pathways related to the previously found HIPARCO-score miRNAs.

Methods: We performed an enrichment analysis using Ingenuity Pathway Analysis. The genes potentially associated with the miRNAs were filtered based on their confidence level. Particularly for cardiovascular disease, only the genes regulated by at least two of the miRNAs were studied.

Results: We observed that the miRNAs studied regulate 200-249 molecules associated with several functions and diseases, including extracranial solid tumors and abdominal neoplasms, among others. The cardiac hypertrophy and NF-kB signaling pathways were identified as the cardiovascular pathways most influenced by these three miRNAs. Conclusions: Considering this, the mechanisms by which CPAP treatment decreases the blood pressure in OSA patients with $\mathrm{RH}$ could be related to these pathways. Further investigations will be necessary to confirm these findings, contributing to the elucidation of new therapeutic targets in patients that do not respond to CPAP treatment.

\section{Background}

Obstructive sleep apnea (OSA) is the most common sleep-disordered breathing, with a prevalence of $20 \%$ in the middle-aged population $(9,29)$. This disorder is caused by the collapse of the upper airway during sleep, which promotes intermittent hypoxemia and hypercapnia. These events are usually accompanied by cortical arousals during the night, leading to daytime somnolence and poor quality of life. The repetitive cycles of upper airway collapse during sleep induce the activation of various pathways related to endothelial dysfunction, oxidative stress, sympathetic activation, inflammation, hypercoagulability, and metabolic dysregulation $(13,22)$. This explains, at least in part, the relationship between OSA and atherosclerosis. Basic research and epidemiological and clinical data support the concept that OSA has a role in the initiation or progression of several cardiovascular diseases $(14,27)$.

Continuous positive airway pressure (CPAP) is the first-choice treatment for sleep apnea. Although CPAP treatment does not demonstrate effectiveness for secondary cardiovascular prevention(17), observational studies show a lower incidence of cardiovascular disease (CVD) in patients treated with CPAP(16). Additionally, treatment with CPAP showed effectiveness in controlling blood pressure in patients with resistant hypertension $(\mathrm{RH})$, which is of extreme importance, considering that increased blood pressure is the main factor for CVD development $(4,8)$. However, despite good CPAP compliance, 
substantial variability exists in its effects. This increases the interest and necessity to develop tools to identify patients who could benefit from this treatment in terms of blood pressure reduction.

MicroRNAs (miRNAs) are small RNAs that inhibit protein-coding messenger RNAs (mRNAs) through translational repression and degradation. These molecules regulate a wide range of biological processes, including development, differentiation, proliferation, metabolism and apoptosis(5). Accordingly, miRNAs are linked with the pathogenesis of human diseases, such as cancer, viral infections, metabolic disorders and cardiovascular diseases. Considering this, miRNAs are interesting diagnostic and prognostic biomarkers $(7,15)$.

Our research group described the existence of a singular cluster of circulating miRNAs that predicts blood pressure responses to CPAP treatment in patients with $\mathrm{RH}$ and OSA(29). This specific expression profile gives a quantitative score parameter, the HIPARCO- score, which identifies those patients with $\mathrm{RH}$ and OSA who are more likely to exhibit a favorable blood pressure response to CPAP. This approach constitutes the first validated precision medicine tool for $\mathrm{RH}$ management in OSA patients. However, the biological mechanisms that underlie this outcome are still unknown. The identification of genes, functions, and pathways related to this molecular signature will improve the understanding of the role of OSA in the pathogenesis of cardiovascular diseases, ultimately benefiting OSA patients with RH who do not present a favorable blood pressure response after CPAP treatment. Considering this, the aim of the present study was to perform a post hoc analysis to investigate the genes, functions and pathways related to the previously found HIPARCO-score miRNAs.

\section{Methods}

\section{Study population and miRNA analysis}

The selection of miRNAs was based on a study by Sánchez-de-la-Torre et al(29). Briefly, the study was conducted with patients diagnosed with RH and OSA from the HIPARCO study (NCT00616265), which was carried out in 24 hospitals in Spain. Patients with RH and OSA who used CPAP for more than 4 hours per night were selected to determine the responder patients in relation to blood pressure (BP) levels and therefore establish whether there was a miRNA profile associated with a positive response to CPAP treatment.

To identify this profile, patients were divided into training and validation sets with two different groups regarding their response to $\mathrm{CPAP}$ treatment: responder (BP change $>4.5 \mathrm{mmHg}$ ) or nonresponder (BP change $<4.5 \mathrm{mmHg}$ ) groups. Human cardiovascular system-specific miRNA arrays (84 miRNAs; Qiagen) were used with the blood plasma of the patients to identify differentially expressed miRNAs. Seven differentially expressed miRNAs were found, of which three miRNAs (miR-378-3p, miR-438-5p, and miR100-5p) were included in a logistic regression model for the training set. These three miRNAs had the highest statistical associations and were linked to an average favorable BP response to CPAP treatment. We simplified the model's use by producing a simple numeric score (the HIPARCO-score, ranging from 0 
to 6) that predicts the probability of a favorable BP response to adherent CPAP use. The model showed high discrimination, with an area under the curve (AUC) of 0.88 . This model was validated in a validation set with an AUC of 0.92. The miRNAs miR-378-3p, miR-438-5p, and miR-100-5p were chosen for in silico analysis to investigate the genes, functions and pathways related to the HIPARCO-score miRNAs.

This study was conducted with permission to use the patients' records and the confidentiality was maintained (CEIC number 48/2008. Consorci Hospital Universitari General de València).

\section{Data analysis}

The overall design and data analysis workflow are presented in Fig. 1. We performed enrichment analysis using Ingenuity Pathway Analysis (IPA) (Ingenuity Systems, Redwood City, CA) to link the miRNAs with their mRNA target genes. This platform provides a literature-curated analysis of networks, biological processes, canonical pathways, diseases and functions associated with miRNAs and target genes. The IPA miRNA Target Filter tool was used to identify the genes regulated by each miRNA. This software identifies a list of potentially regulated genes based on four databases (TargetScan, miRecords, Ingenuity Knowledge Base and TarBase). The genes potentially associated with the miRNAs were filtered based on their confidence level, and only experimentally observed and highly predicted target genes were used in the analysis (Fig. 4).

The associations between the predicted miRNA target genes and networks, canonical pathways, diseases and functions were analyzed. We first established the mRNAs regulated by these miRNAs, and then, from the list of mRNAs/genes, the canonical pathways were examined. Different diseases and associated pathways were also explored, especially cardiovascular-related ones (for this, only the genes regulated by at least two of the miRNAs were studied).

\section{Results}

\section{Canonical pathway analysis}

The HIPARCO-score molecular signature comprises three miRNAs (miR-378a-3p, miR-100-5p, and miR486-5p). The diseases and functions most significantly associated with these miRNAs are summarized in Table 1. The studied miRNAs regulate 200-249 molecules in each disease or function, including digestive organ tumors, extracranial solid tumors, abdominal neoplasms, nonhematologic cancers, solid tumors, and carcinomas. 
Table 1

The diseases/functions and canonical pathways regulated by the studied miRNAs.

\begin{tabular}{|lll|}
\hline Diseases or functions & p-value & Molecules \\
\hline Digestive organ tumor & $6.02 \mathrm{E}-29$ & 234 \\
\hline Extracranial solid tumor & $1.12 \mathrm{E}-27$ & 248 \\
\hline Abdominal neoplasm & $2.05 \mathrm{E}-27$ & 238 \\
\hline Nonhematologic malignant neoplasm & $1.17 \mathrm{E}-26$ & 244 \\
\hline Solid tumor & $6.11 \mathrm{E}-25$ & 249 \\
\hline Carcinoma & $2.22 \mathrm{E}-23$ & 227 \\
\hline Tumorigenesis of tissue & $5.60 \mathrm{E}-25$ & 229 \\
\hline Cancer & $1.67 \mathrm{E}-22$ & 245 \\
\hline Adenocarcinoma & $1.43 \mathrm{E}-21$ & 209 \\
\hline Abdominal cancer & $6.07 \mathrm{E}-21$ & 221 \\
\hline Pathways & Genes & \\
\hline Axonal Guidance Signaling & 11 & \\
\hline Glucocorticoid Receptor Signaling & 10 & \\
\hline Molecular Mechanisms of Cancer & 10 & \\
\hline Role of Macrophages and Fibroblasts & 10 & \\
\hline Glioblastoma Multiforme Signaling & 9 & \\
\hline Human Embryonic Stem Cell Pluripotency & 9 & \\
\hline Xenobiotic Metabolism Signaling & 9 & \\
\hline AMPK Signaling & 9 & \\
\hline Role of Osteoblast and Osteoclasts & 9 & \\
\hline Glioma Signaling & 8 & \\
\hline
\end{tabular}

Regarding the canonical pathways (Table 1), we observed that these miRNAs target 8-11 genes in each of the processes in which they are involved. In axonal guidance signaling, for example, 11 genes are targeted by these miRNAs, while 10 genes are targeted in glucocorticoid receptor signaling and molecular mechanisms of cancer.

\section{Cardiovascular-targeted canonical pathway analysis}


An in-depth study was carried out to investigate the cardiovascular-related functions and canonical pathways significantly altered by at least two of the miRNAs studied (Table 2). The major cardiovascular functions associated with these miRNAs are related to the development of blood vessels and cardiovascular tissues. We observed 30, 26 and 18 molecules altered in angiogenesis, vasculogenesis and cardiogenesis, respectively ( $p$-value $<0.05$ ).

Table 2

Cardiovascular diseases and functions regulated by the studied miRNAs.

\begin{tabular}{|lll|}
\hline Diseases and functions & p-value & Molecules \\
\hline Angiogenesis & $7.57 \mathrm{E}-04$ & 30 \\
\hline Vasculogenesis & $5.79 \mathrm{E}-04$ & 26 \\
\hline Cardiogenesis & $2.27 \mathrm{E}-04$ & 18 \\
\hline Hypertrophy of heart & $3.51 \mathrm{E}-02$ & 10 \\
\hline Heart failure & $2.44 \mathrm{E}-02$ & 10 \\
\hline Development of artery & $1.92 \mathrm{E}-04$ & 8 \\
\hline Apoptosis of heart & $3.45 \mathrm{E}-02$ & 7 \\
\hline Formation of coronary vessel & $1.76 \mathrm{E}-05$ & 7 \\
\hline Hypertrophy of cardiac muscle & $2.24 \mathrm{E}-02$ & 6 \\
\hline Formation of heart tissue & $3.05 \mathrm{E}-04$ & 6 \\
\hline Proliferation of heart cells & $2.63 \mathrm{E}-02$ & 4 \\
\hline Blood vessel collateralization of coronary artery & $7.77 \mathrm{E}-04$ & 4 \\
\hline Development of atrioventricular valve & $5.53 \mathrm{E}-04$ & 3 \\
\hline Hypoplasia of myocardium & $7.20 \mathrm{E}-03$ & 2 \\
\hline Injury of heart tissue & $1.71 \mathrm{E}-02$ & 1 \\
\hline Injury of left ventricle & $8.61 \mathrm{E}-03$ & 1 \\
\hline Hypoplasia of ventricular compact zone & $8.61 \mathrm{E}-03$ & 1 \\
\hline Oxidative stress response of cardiomyocytes & $4.23 \mathrm{E}-02$ & 1 \\
\hline Atresia of tricuspid valve & $3.40 \mathrm{E}-02$ & 1 \\
\hline Experimentally induced heart failure & $1.71 \mathrm{E}-02$ & 1 \\
\hline Thrombosis of glomerular capillary & $4.23 \mathrm{E}-02$ & 1 \\
\hline
\end{tabular}

To focus on the most representative canonical pathways related to cardiovascular disease, we studied the cardiac hypertrophy and nuclear factor kappa beta (NF-kB) signaling canonical pathways. In the 
cardiac hypertrophy signaling canonical pathway, miR-100 regulates the expression of mammalian target of rapamycin (mTOR), and together with miR-378a, it regulates insulin-like growth factor 1 (IGF1R). miR378a-3p also affects the expression of the growth factor receptor-bound protein 2 (GRB2) and interleukin 6 receptor (IL-6R). Moreover, miR-486 interferes with the regulation of the expression of eukaryotic translation initiation factor 4E (elF4E), p70S6 kinase (p70S6K) and the complex TAK1/TAB1 (Fig. 1). The three miRNAs also participate in the regulation of nuclear factor of activated T-cells (NFAT). miR-100-5p and miR-378a-3p jointly regulate the expression of leukemia inhibitory factor (LIF) and IGF1R, while miR486 regulates transforming growth factor- $\beta$-activated kinase 1 (TAK1). In addition, miR-378a-3p alters the expression of the glycoprotein 130 (GP130) dimer and SHC-GRB2-SOS complex (Fig. 2).

Regarding the NF-kB signaling canonical pathway, miR-486-5p and miR-378a-3p regulate the expression of TAK1 and tumor necrosis factor receptor-associated factor 6 (TRAF6), respectively, leading to an alteration in the activation of the NF-kB pathway (Fig. 3). This ultimately leads to an alteration in the mechanisms of cell survival and cell proliferation.

\section{Discussion}

In the present study, we performed an enrichment analysis using IPA to observe the pathways regulated by the HIPARCO-score miRNAs (miR-378a-3p, miR-100-5p and miR-486-5p), a set of miRNAs that identify patients more likely to exhibit a favorable BP response to CPAP. Our main objective was to investigate the main biological functions regulated by these three miRNAs that are involved in the favorable response to CPAP treatment in patients with OSA and $\mathrm{RH}$.

We observed the contribution of miR-378a-3p, miR-100-5p and miR-486-5p in the regulation of a variety of genes and pathways, especially in those related to cardiovascular function and cancer. Specifically, we observed a role for these miRNAs in heart hypertrophy, heart failure, apoptosis, and heart tissue injury. This finding is corroborated by a variety of studies in the literature demonstrating an association between miRNAs and cardiovascular disease and hypertension $(18,25)$. Similarly, studies have shown a strong relation between OSA and these events. Therefore, it has been reported that OSA is associated with hypertension and with hypertension-associated end-stage organ diseases such as stroke, coronary heart disease, and arrhythmia(33). Moreover, previous studies suggest that OSA has a role in the initiation and progression of several cardiovascular diseases(28). Considering this, the effect of CPAP in RH patients could be mediated by these miRNAs through their actions on cardiovascular-associated genes and functions. In fact, we observed that the studied miRNAs are associated with the NF-kB signaling pathway, which is known to upregulate the transcription of genes involved in endothelin signaling(23). Accordingly, several NF-kB binding sites were identified in the endothelin gene promoter region(10). Thus, the mechanism by which CPAP treatment decreases BP may be associated with the involvement of these miRNAs with the NF-kB signaling pathway, ultimately affecting the endothelin signaling. Further studies will be necessary to confirm this. 
Cardiac failure is often associated with prolonged and maladaptive cardiac hypertrophy $(11,21)$. Considering that cardiomyocytes lose their ability to divide soon after birth, cardiac hypertrophy is an important adaptive response to maintain or increase the cardiac output of the organism(19). We observed that miR-378a-3p, miR-100-5p and miR-486-5p are involved in the regulation of several molecules related to activation pathways that lead to a hypertrophic response at the cellular level. For instance, the miR-378a-3p-mediated induction of the cytokine receptor GP130 activates the MAPK, PI3K and signal transducer and activator of transcription 3 (STAT3) pathways, which leads to the activation of genes involved in hypertrophy and survival pathways(32). Consequently, the translation of various signals through the GRB2-SOS complex and Akt activation that targets mTOR also occurs. mTOR speeds up the process of protein synthesis by activating its downstream targets p70S6K and elF4E, resulting in protein synthesis, cell growth and cell proliferation. In turn, these kinases mediate cellular responses to stress, such as DNA damage and nutrient deprivation(26). Interestingly, Wen et al demonstrated that intermittent hypoxia, which is one of the main features of OSA, led to the inhibition of mTOR phosphorylation and activation of AMPK phosphorylation, inducing cellular apoptosis(31). On the other hand, a variety of studies reported an association between cardiac hypertrophy and the severity of OSA(20). Considering these findings, it is possible to observe the role of the studied miRNAs in the cardiovascular-related pathways that are also affected by OSA. In addition, it is important to address that $\mathrm{RH}$ may lead to cardiac hypertrophy(6). Thus, the studied miRNAs associated with a favorable response to CPAP treatment could be involved in the regulation of such adverse outcome in the long-term. This highlights the importance of these miRNAs not only as biomarkers but also as possible therapeutic targets. This relationship should be further explored in future studies.

miR-378a-3p, miR-100-5p and miR-486-5p also seem to regulate the effect of proinflammatory cytokines in beta cells via NF-kB $(2,24)$. miR-378a regulates TAK1, a kinase that mediates the signal transduction induced by TGF-beta and bone morphogenetic protein (BMP), and controls a variety of cell functions, including transcription regulation and apoptosis. Additionally, in response to interleukin-1 (IL-1), TAK1 forms a kinase complex that includes TRAF6 (also regulated by miR-486), MAP3K7P1/TAB1 and MAP3K7P2/TAB2; this complex is required for the activation of NF-kB(1). Therefore, these miRNAs modify NF-kB activity, which is pivotal for activating or preventing the overstimulation of the toll-like receptor (TLR) pathway(2). On the other hand, rapid reoxygenation at the end of apneas/hypopneas leads to the production of free radicals, inducing oxidative stress and the upregulation of NF-kB. In addition, there is evidence indicating that CPAP treatment reduces the levels of inflammatory mediators, such as interleukin-6, tumor necrosis factor-a, and C-reactive protein(3). Thus, there is considerable compatibility between the molecules and pathways affected by the studied miRNAs and those affected by OSA or CPAP treatment regarding inflammatory mechanisms.

A substantial number of studies have reported the oncogenic and tumor suppressing roles of miRNAs. Additionally, several miRNAs are markers for the early diagnosis of cancer(12,30). In fact, the present study suggests a role for the studied miRNAs in a variety of cancer-related pathways. This leads to a possibility for which there is still no evidence that the phenotype of good CPAP responders in terms of BP might be associated with other characteristics, such as different prognosis in tumor-related diseases. 
It is important to note that there are still considerable limitations in miRNA studies, and more investigations are needed to confirm the role of miR-378a-3p, miR-100-5p, and miR-486-5p in cardiovascular diseases. Considering that these miRNAs were measured in plasma, no distinction was made between miRNAs derived from exosomes and those derived from other events, such as cell death. Thus, the results discussed herein should be interpreted with caution. Regardless, the observed significant change in the circulating profile of miRNAs is sufficient as a biomarker to be interpreted by itself.

\section{Conclusions}

We observed the contribution of miR-378a-3p, miR-100-5p and miR-486-5p in the regulation of a variety of genes and pathways, especially in those related to cardiovascular function and cancer. The cardiac hypertrophy and NF-kB signaling pathways were identified as the cardiovascular pathways most influenced by these three miRNAs. Considering this, the mechanisms by which CPAP treatment decreases the blood pressure in OSA patients with $\mathrm{RH}$ could be related to these pathways. Further investigations in different populations, as well as an evaluation of their functional impacts on putative target genes and pathways will be necessary to confirm these findings. Improved knowledge of the pathways altered by these 3 miRNAs will ultimately lead to a better understanding of the molecular mechanisms underlying lowered BP levels after CPAP treatment. In addition, this will contribute to the elucidation of new therapeutic targets in patients that do not respond to CPAP treatment.

\section{List Of Abbreviations}

AUC: Area under the curve

BMP: Bone morphogenetic protein

BP: Blood pressure

CPAP: Continuous positive airway pressure

elF4E: Eukaryotic translation initiation factor 4E

GP130: Glycoprotein 130

GRB2: Growth factor receptor-bound protein 2

IGF1R: Insulin-like growth factor 1

IL-1: Interleukin-1

IL-6R: Interleukin 6 receptor

IPA: Ingenuity Pathways Analysis 
LIF: Leukemia inhibitory factor

MAP3K7P1/TAB1: Mitogen-activated protein kinase kinase kinase 7-interacting protein 1/TAK binding protein 1

MAP3K7P2/TAB2: Mitogen-activated protein kinase kinase kinase 7-interacting protein 2/TAK binding protein 2

miRNA: MicroRNA

mRNAs: Messenger RNAs

mTOR: Mammalian target of rapamycin

NFAT: Nuclear factor of activated T-cells

NF-kB: Nuclear factor kappa beta

OSA: Obstructive sleep apnea

p70S6K: p70S6 kinase

$\mathrm{RH}$ : Resistant hypertension

SHC: SH2-containing collagen-related proteins

SOS: Son of sevenless

STAT3: Signal transducer and activator of transcription 3

TAK1: Transforming growth factor- $\beta$-activated kinase 1

TLR: Toll-like Receptor

TRAF6: Tumor necrosis factor receptor-associated factor 6

\section{Declarations}

Ethics approval and consent to participate: The HIPARCO study was approved by the ethic commitee of each participating center (02/05/08_48) and all patients provided written informed consent.

Consent for publication: Not applicable

Availability of data and material: Not applicable

Competing interests: None 
Funding: Supported by: ISCIII (PI14/01266) (PI18/00449), co-funded by FEDER “Una manera de hacer Europa", the Spanish Respiratory Society (SEPAR), and the Associació Lleidatana de Respiratori (ALLER).

Authors' contributions: AZ, FS, FB and MS contributed to the study concept and design; AZ, FS, AS, IB, MAM, FB and MS contributed to the data acquisition; AZ, FS, AT, LP, AS, FB and MS contributed to the data analysis and interpretation; and AZ, FS, AT, LP, IB, AS, MAM, FB and MS contributed to the manuscript draft, critically revised the manuscript for important intellectual content and approved the final version. MS is the guarantor of the paper.

Acknowledgments: This study was supported by Instituto de Salud Carlos III (PI14/01266, PI18/00449), Fondos FEDER "Una manera de hacer europa", the Spanish Respiratory Society (SEPAR), and the Associació Lleidatana de Respiratori (ALLER).

\section{References}

1. Akira S, Takeda K. Toll-like receptor signalling. Nat Rev Immunol 2014; 4: 499-511.

2. Assmann TS, Recamonde-Mendoza M, De Souza BM, Crispim D. MicroRNA expression profiles and type 1 diabetes mellitus: systematic review and bioinformatic analysis. Endocr Connect 2017;6: $773-790$

\section{Baessler A, Nadeem R, Harvey M, Madbouly E, Younus A, Sajid H, Naseem J, Asif A, Bawaadam H.} Treatment for sleep apnea by continuous positive airway pressure improves levels of inflammatory markers - a meta-analysis. J Inflamm 2013; 10: 13..

4. Barceló A, Piérola J, De La Peña M, Esquinas C, Fuster A, Sanchez-de-la-Torre M, Carrera M, AlonsoFernandez A, Ladaria A, Bosch M, Barbé F. Free fatty acids and the metabolic syndrome in patients with obstructive sleep apnoea. Eur. Respir. J. 2011; doi: 10.1183/09031936.00050410.

5. Bartel DP. MicroRNAs: Genomics, Biogenesis, Mechanism, and Function. Cell: 2004; doi: 10.1016/s0092-8674(04)00045-5

6. Blaustein MP. How does pressure overload cause cardiac hypertrophy and dysfunction? Highouabain affinity cardiac Na + pumps are crucial . Am. J. Physiol. Circ. Physiol. 2017; doi: 10.1152/ajpheart.00131.2017.

7. Christopher A, Kaur R, Kaur G, Kaur A, Gupta V, Bansal P. MicroRNA therapeutics: Discovering novel targets and developing specific therapy. Perspect. Clin. Res. 2016; doi: 10.4103/2229-3485.179431.

8. Durán-Cantolla J, Aizpuru F, Montserrat JM, Ballester E, Terán-Santos J, Aguirregomoscorta Jl, Gonzalez M, Lloberes P, Masa JF, De La Peña M, Carrizo S, Mayos M, Barbé F. Continuous positive airway pressure as treatment for systemic hypertension in people with obstructive sleep apnoea: Randomised controlled trial. BMJ 2010; doi: 10.1136/bmj.c5991.

9. Durán J, Esnaola S, Rubio R, Iztueta A. Obstructive sleep apnea-hypopnea and related clinical features in a population-based sample of subjects aged 30 to 70 yr. Am J Respir Crit Care Med 163: 685-9, 2001. 
10. Fomin V, Richard P, Hoque M, Li C, Gu Z, Fissore-O'Leary M, Tian B, Prives C, Manley JL. The C90RF72 Gene, Implicated in Amyotrophic Lateral Sclerosis and Frontotemporal Dementia, Encodes a Protein That Functions in Control of Endothelin and Glutamate Signaling . Mol. Cell. Biol. 2018; doi: 10.1128/mcb.00155-18.

11. Frey N, Olson EN. Cardiac Hypertrophy: The Good, the Bad, and the Ugly. Annu. Rev. Physiol. 2003; doi: 10.1146/annurev.physiol.65.092101.142243.

12. Hosseinahli N, Aghapour M, Duijf PHG, Baradaran B. Treating cancer with microRNA replacement therapy: A literature review. J. Cell. Physiol.: 2018; doi: 10.1002/jcp.26514

13. Lavie L. Oxidative stress in obstructive sleep apnea and intermittent hypoxia - Revisited - The bad ugly and good: Implications to the heart and brain. Sleep Med. Rev.: 2015; doi:

10.1016/j.smrv.2014.07.003.

14. Lee CH, Sethi R, Li R, Ho HH, Hein T, Jim MH, Loo G, Koo CY, Gao XF, Chandra S, Yang XX, Furlan SF, Ge Z, Mundhekar A, Zhang WW, Uchôa CHG, Kharwar RB, Chan PF, Chen SL, Chan MY, Richards AM, Tan HC, Ong TH, Roldan G, Tai BC, Drager LF, Zhang JJ. Obstructive sleep apnea and cardiovascular events after percutaneous coronary intervention. Circulation 2016; doi:

10.1161/CIRCULATIONAHA.115.019392.

15. Li M, Zhang J. Circulating MicroRNAs: Potential and Emerging Biomarkers for Diagnosis of Cardiovascular and Cerebrovascular Diseases. Biomed Res. Int. 2015; doi: 10.1155/2015/730535.

16. Marin JM, Agusti A, Villar I, Forner M, Nieto D, Carrizo SJ, Barbé F, Vicente E, Wei Y, Javier Nieto F, Jelic S. Association between treated and untreated obstructive sleep apnea and risk of hypertension. JAMA - J. Am. Med. Assoc. 2012; doi: 10.1001/jama.2012.3418.

17. McEvoy RD, Antic NA, Heeley E, Luo Y, Ou Q, Zhang X, Mediano O, Chen R, Drager LF, Liu Z, Chen G, Du B, McArdle N, Mukherjee S, Tripathi M, Billot L, Li Q, Lorenzi-Filho G, Barbe F, Redline S, Wang J, Arima H, Neal B, White DP, Grunstein RR, Zhong N, Anderson CS, SAVE Investigators and Coordinators. CPAP for Prevention of Cardiovascular Events in Obstructive Sleep Apnea. N Engl J Med 2016; 375: 919-931.

18. Navickas R, Gal D, Laucevičius A, Taparauskaite A, Zdanyte M, Holvoet P. Identifying circulating microRNAs as biomarkers of cardiovascular disease: A systematic review. Cardiovasc. Res. 2016; doi: $10.1093 / \mathrm{cvr} / \mathrm{crw} 174$.

19. Nie X, Fan J, Li H, Yin Z, Zhao Y, Dai B, Dong N, Chen C, Wang DW. miR-217 Promotes Cardiac Hypertrophy and Dysfunction by Targeting PTEN. 2018; doi: 10.1016/j.omtn.2018.05.013.

20. Noda A, Okada T, Yasuma F, Nakashima N, Yokota M. Cardiac hypertrophy in obstructive sleep apnea syndrome. Chest 1995; doi: 10.1378/chest.107.6.1538.

21. Pauly DF. Cardiac function and heart failure. In: Journal of the American College of Cardiology. 2005; doi: 10.1016/j.jacc.2005.05.005

22. Phillips CL, McEwen BJ, Morel-Kopp MC, Yee BJ, Sullivan DR, Ward CM, Tofler GH, Grunstein RR. Effects of continuous positive airway pressure on coagulability in obstructive sleep apnoea: A randomised, placebo-controlled crossover study. Thorax 2012; doi: 10.1136/thoraxjnl-2011-200874. 
23. Quehenberger $P$, Bierhaus A, Fasching P, Muellner C, Klevesath $M$, Hong M, Stier G, Sattler M, Schleicher E, Speiser W, Nawroth PP. Endothelin 1 transciption is controlled by nuclear factor-KB in AGE-stimulated cultured endothelial cells. Diabetes 2000; doi: 10.2337/diabetes.49.9.1561.

24. Roggli E, Britan A, Gattesco S, Lin-Marq N, Abderrahmani A, Meda P, Regazzi R. Involvement of MicroRNAs in the Cytotoxic Effects Exerted by Proinflammatory Cytokines on Pancreatic -Cells. Diabetes 2010; 59: 978-986.

25. Romaine SPR, Charchar FJ, Samani NJ, Tomaszewski M. Circulating microRNAs and hypertension From new insights into blood pressure regulation to biomarkers of cardiovascular risk. Curr. Opin. Pharmacol. 2016; :1-7.

26. Samidurai A, Kukreja RC, Das A. Emerging Role of mTOR Signaling-Related miRNAs in Cardiovascular Diseases. Oxid Med Cell Longev 2018; 6141902.

27. Sánchez-de-la-Torre M, Barbé F. Medicina personalizada en apnea del sueño: hacia un nuevo paradigma de tratamiento integral de la enfermedad. Med. Clin. (Barc). 2016; doi:

10.1016/j.medcli.2016.09.002.

28. Sánchez-de-la-Torre M, Campos-Rodriguez F, Barbé F. Obstructive sleep apnoea and cardiovascular disease. Lancet Respir. Med.: 2013;1:61-72.

29. Sánchez-de-la-Torre M, Khalyfa A, Sánchez-de-la-Torre A, Martinez-Alonso M, Martinez-García MÁ, Barceló A, Lloberes P, Campos-Rodriguez F, Capote F, Diaz-de-Atauri MJ, Somoza M, González M, Masa J-F, Gozal D, Barbé F, Spanish Sleep Network. Precision Medicine in Patients With Resistant Hypertension and Obstructive Sleep Apnea. J Am Coll Cardio/ 2015; 66: 1023-1032.

30. Vannini I, Fanini F, Fabbri M. Emerging roles of microRNAs in cancer. Curr Opin Genet Dev. 2018;48:128-133.

31. Wen Z, Liang D, Cai X, Chen J. The role of AMPK / mTOR signal pathway in brain injury following chronic intermittent hypoxia in growing rats. Eur Rev Med Pharmacol Sci. 2018; 22:1071-1077.

32. Yasukawa H, Hoshijima M, Gu Y, Nakamura T, Pradervand S, Hanada T, Hanakawa Y, Yoshimura A, Ross J, Chien KR, Chien KR. Suppressor of cytokine signaling-3 is a biomechanical stress-inducible gene that suppresses gp130-mediated cardiac myocyte hypertrophy and survival pathways. J Clin Invest 2001; 108: 1459-67,.

33. Zhang W, Si LY. Obstructive sleep apnea syndrome (OSAS) and hypertension: Pathogenic mechanisms and possible therapeutic approaches. Ups J Med Sci 2012;117: 370-382.

\section{Figures}




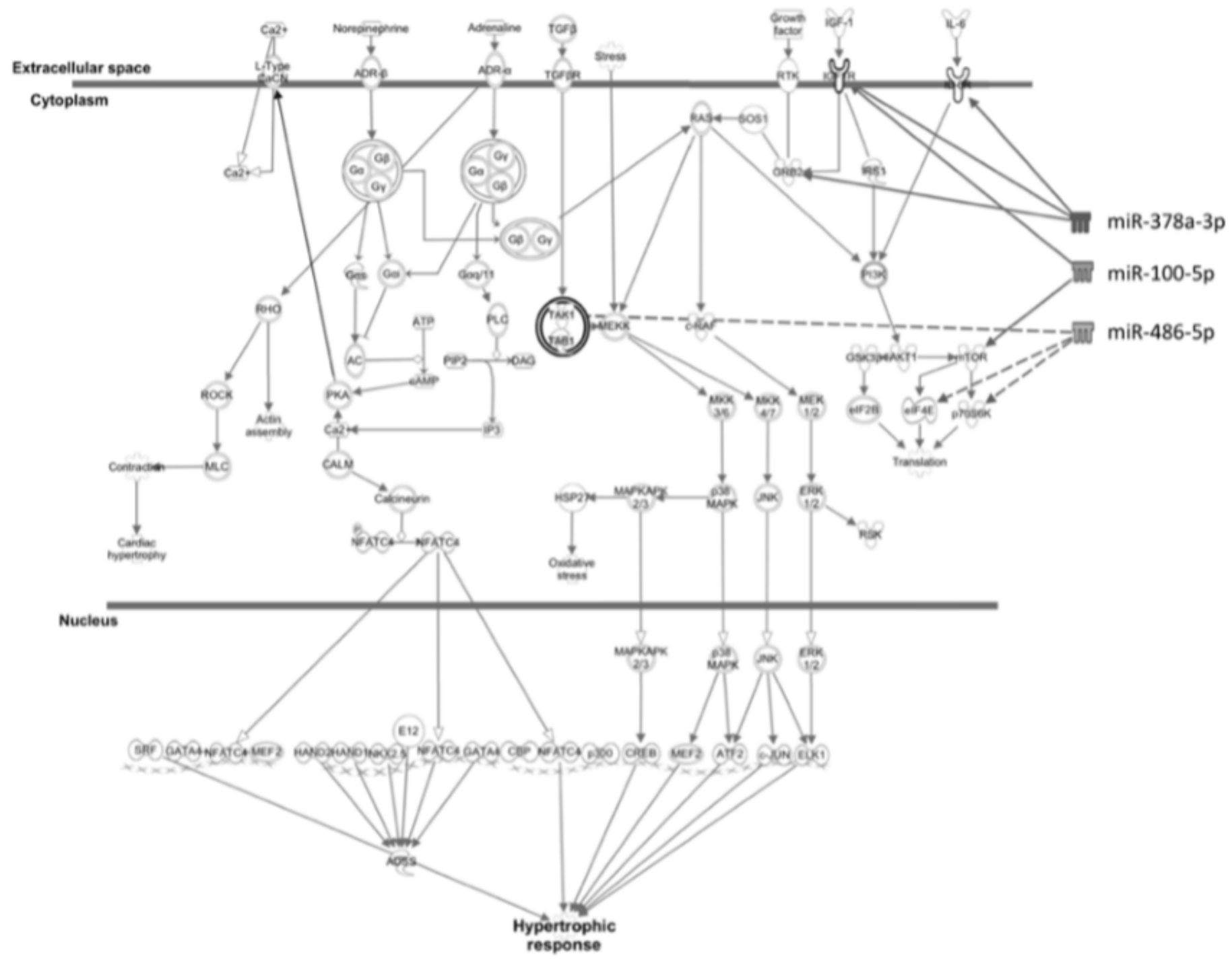

Figure 1

miRNA target genes in the cardiac hypertrophy signaling canonical pathway: miR-100-5p directly regulates mTOR. Additionally, miR-100-5p and miR-378a-3p directly modulate the IGF1 membrane receptor. miR-378a-3p also regulates the membrane receptor of IL-6 and GRB2 at the cytoplasmic level. miR-486-5p has an indirect effect on elF4E, p70S6K and on the TAK1/TAB1 complex. 
miR-100

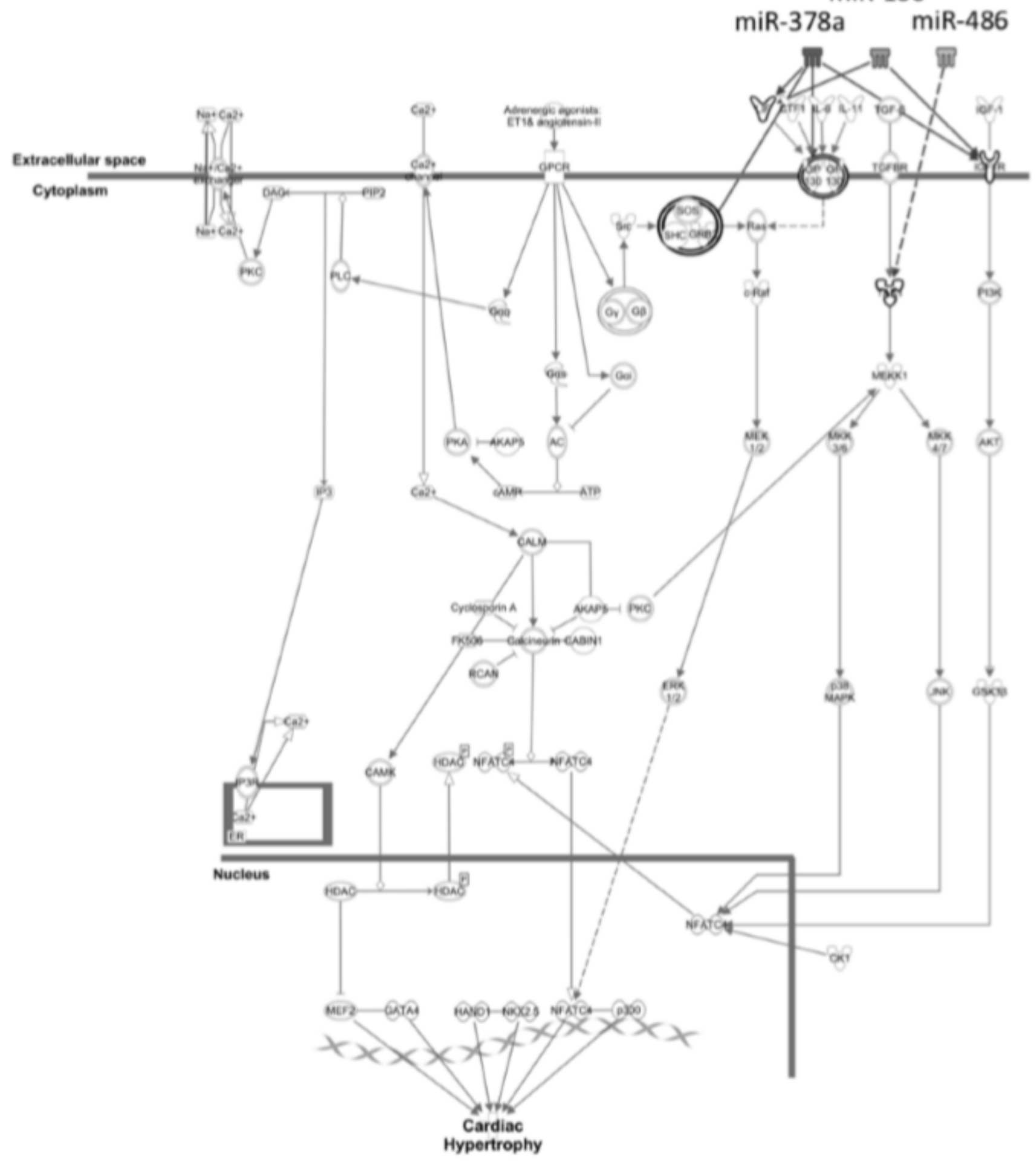

\section{Figure 2}

miRNA target genes in the NFAT pathway: miR-100-5p and miR-378a-3p directly regulate the IGF1 membrane receptor and LIF. miR-378a-3p acts on the formation of the SCH-GRB2-SOS complex at the cytoplasmic level. In addition, this miRNA is directly involved in the activity of the GP130 dimer. miR-486$5 p$ is indirectly implicated in the regulation of TAK1. 


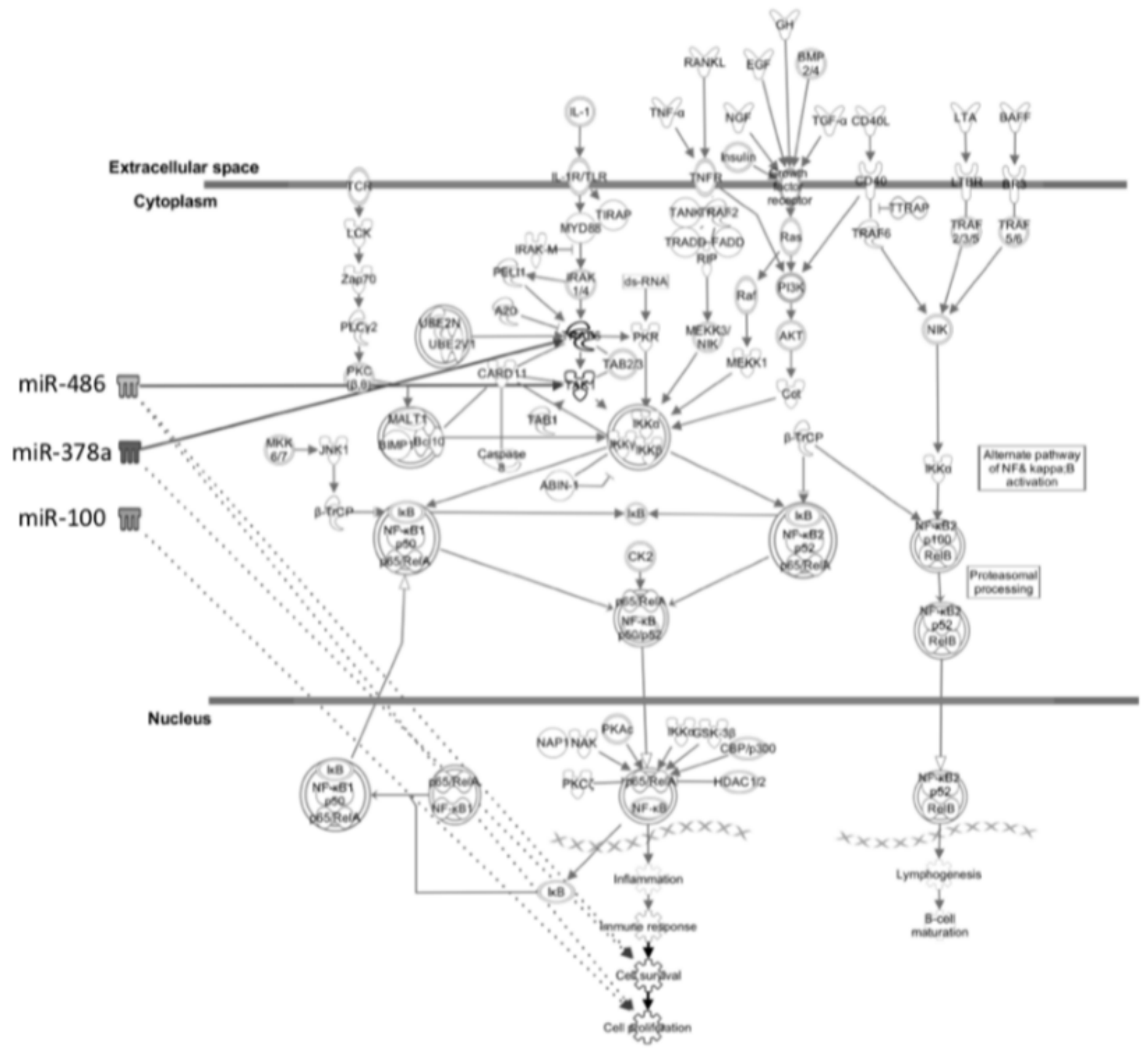

Figure 3

miRNA target genes in the NF-kB signaling canonical pathway: miR-486-5p directly regulates TAK1, and miR-378a-3p directly modulates TRAF6 at the cytoplasmic level. miR-486-5p, miR-378a-3p and miR-100$5 p$ indirectly alter the functions of cellular survival and proliferation. 


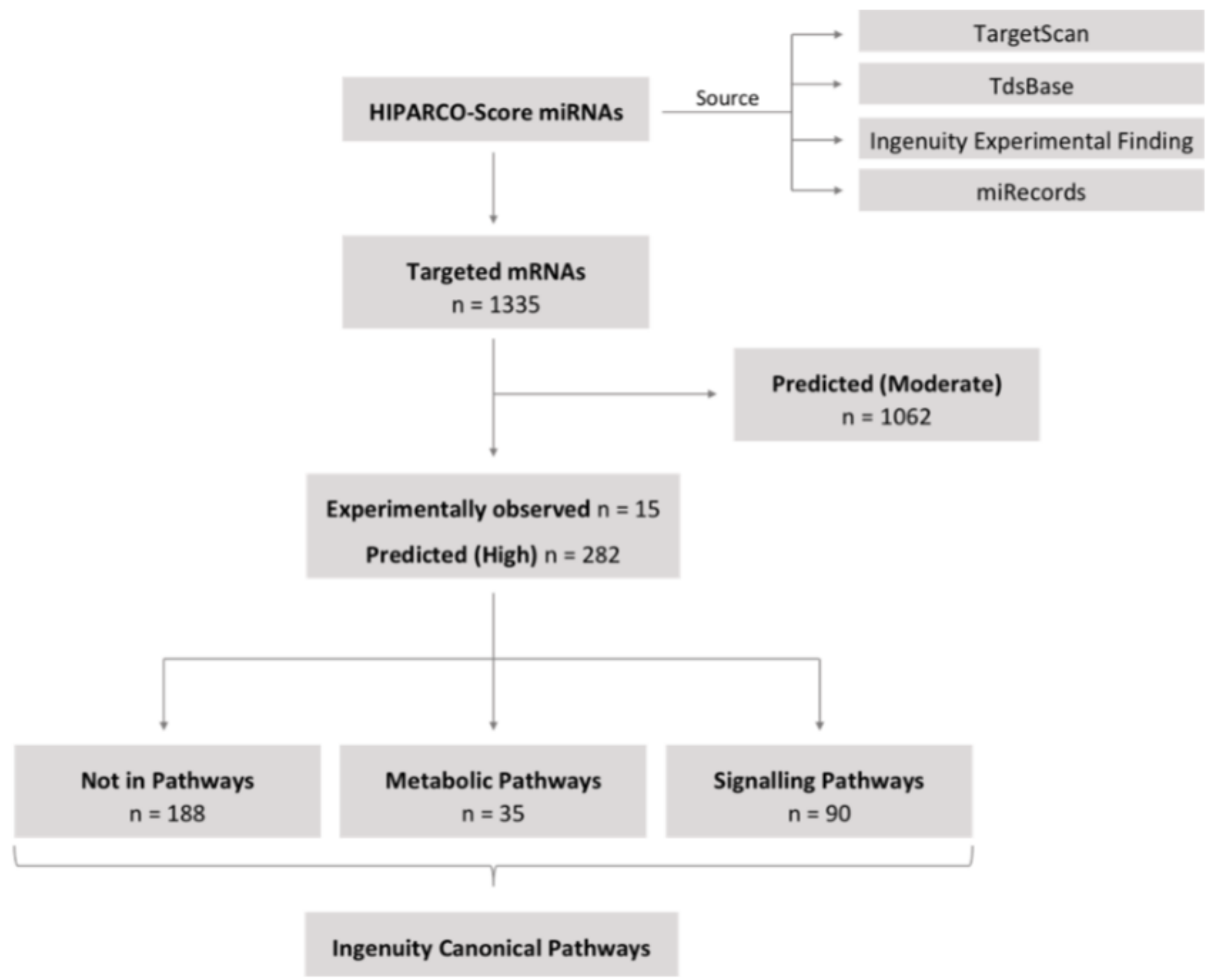

Figure 4

Flowchart of the study 